\title{
Prospects of Neuroscientific Approaches to the Problem of Consciousness in the Situation of Growing Global Crisis of Our Terrestrial Civilization
}

\author{
David I Dubrovsky* \\ Department of Theory of Knowledge, Institute of Philosophy of the Russian Academy of Sciences, Russia
}

*Corresponding author: David I Dubrovsky, Department of Theory of Knowledge, Institute of Philosophy of the Russian Academy

of Sciences, Moscow, Russia

\section{ARTICLE INFO}

Received: 幽 September 03, 2019

Published: 慧 September 09, 2019

Citation: David I Dubrovsky. Prospects of Neuroscientific Approaches to the Problem of Consciousness in the Situation of Growing Global Crisis of Our Terrestrial Civilization. Biomed J Sci \& Tech Res 21(2)-2019. BJSTR. MS.ID.003565.

\section{ABSTRACT}

Human activity is determined by his consciousness, his values, goals and decisions. This activity has led to a global crisis of our consumer civilization, threatening her death. Here the main role is played by such negative properties of consciousness as insatiable consumerism, aggressiveness and excessive egoism. The article discusses debatable issues about the possibility of changing these negative properties during the development of science and technology. The importance of neuroscientific studies of consciousness is examined, especially the study of those brain functions and information processes on which a person's ability to control his consciousness and change its persistent dispositional structures depends. The theoretical and practical difficulties of solving such problems are clarified. The question is whether there is a real possibility of their solution? There is no definite answer to this question in the scientific community yet. But we must maintain an optimistic perspective.

Keywords: Global Crisis; Consciousness; Neuroscience Subjective Reality and the Brain; Information Approach; Mental Causation; Mind Control

\section{Introduction}

Our consumer civilization is experiencing a global crisis that is steadily deepening, leading to degradation and destruction. This is constantly discussed. But on the part of the world's political and economic leaders we do not see any decisive opposition. Negative processes deepen, covering not only ecological and various socioeconomic components of the global crisis, but already clearly demonstrate grave physical changes on our planet [1,2]. Now, more than ever before in the history of mankind, the preservation of faith in human dignity, in the altruistic potential of human nature is urgently needed. The issue of consciousness is a key point in overcoming the global crisis of terrestrial civilization. This is the problem of overcoming negative properties of consciousness such as irrepressible consumerism, aggressiveness and extreme egoism. They are rooted in human nature, and this determines their persistence, immutability at all stages of human history. They have a genetic basis, and this determines the extreme difficulties of changing them. Many doubt very much whether this is possible. But if we fail to do this, the fate of our civilization is deplorable. One thing is clear: this cannot be achieved by means of religion, ideology, upbringing and education alone. For this, new achievements of science and technology are needed. A significant role here belongs to neuroscience and related fields of scientific knowledge.

In recent decades, neuroscience has achieved significant results in the study of consciousness. They are associated, in particular with the use of methods for visualizing brain processes and the removal of signals from individual neurons, discovery and study of mirror neurons, the successful development of the trend called Brain-Reading, with the determination of clear neurodynamic correlates of many psychic phenomena that serve as a foundation for neurotechnologies in medicine and for other practical purposes. 
Neuroscientific development of the problem of consciousness is interdisciplinary. Neurophysiological studies are closely bond to the use of methods and results of many related disciplines such as psychology, psychiatry, psychophysiology, psychopharmacology, psychogenetics, neurosurgery, psychoneurology (the results of the latter are especially important for complex studies of the relation of various states of consciousness to the functioning of certain brain structures; in this respect especially indicative and valuable are the outstanding works of Ramachandran [3]. But very important part belongs, of course, to those disciplines of philosophical, psychological, phenomenological and humanitarian plan that describe consciousness - its meaningful dynamic forms, value-andsemantics, active-and-volitional structures. Without such definite description it is impossible to conduct a neuroscientific study and to explain consciousness. Numerous theoretical and methodological questions arise here on the solution of which the productivity of all this scientific work depends.

First of all, it is necessary to solve most difficult theoretical problem about the nature of the connection between the phenomena of consciousness and brain processes. The problem is that the phenomena of consciousness is described in terms of content, meaning, purpose, faith, will, while brain processes-in terms of the nerve substrate and pulse, mass, energy and spatial properties. These are two different description languages, and there are no direct logical connections between them. In analytical philosophy, they say there is a «gap in explanation» here. In order to relate these two types of descriptions, it is necessary to create a "conceptual bridge", i.e. to find or create such a theoretical system that would be logically relevant to each of the two different ways of describing and could link them in a single theoretical explanation.

But in order to overcome the "gap in explanation", it is necessary to solve not only this epistemological problem, but also the main ontological problem of the relation between the events of subjective reality (to which physical properties cannot be attributed) and the brain processes (which necessarily possess these properties). This issue has always been a stumbling block for natural sciences. Meanwhile, subjective reality is a necessary and a specific property of consciousness, without it there is no consciousness. Neuroscientific research and explanation of this quality compose the essence of what many philosophers call the "Hard problem of consciousness". For more than 60 years it has been the focus of analytical philosophy. Despite the huge amount of publications (over one thousand books and many thousands of articles), no acceptable result has been achieved in its development. It was noted by both Western and Russian philosophers and representatives of neuroscience [4]. The proposed concepts were in general reductionist; thus the specificity of subjective reality was lost.

In fact, to solve this problem it is necessary to give a theoretically correct answer to one main question: how is mental, the phenomenon of subjective reality, connected with brain processes.
This will help to explain the mental causality, arbitrary action, the phenomenon of free will and a number of other difficult questions. Trying to solve this problem, for many years I have been developing an informational approach based on the ideas of evolution and self-organization. The basic theses of the theory developed by me were expounded in my book many years ago [5]. The theory I proposed is different from the existing concepts of analytical philosophy. It relies on three initial premises from which answers to the main questions of the problem are logically derived. The first two premises are generally accepted scientific principles. The third premise is an intuitively acceptable agreement, well confirmed by ordinary (every-day) and scientific experience. I give them below:

a) Information is necessarily embodied in its physical bearer.

b) Information is invariant to the physical properties of its bearer; i.e., the same information can be implemented and transferred by bearers that are different in their physical properties; in other words, the same information can be coded in different ways.

c) A phenomenon of subjective reality, for example, my visual image of a given object experienced in a given interval (let us designate by A) can be regarded as information about this subject.

It is worth noting that information in biological and social systems allows not only a syntactic description, but also a semantic (meaningful) and pragmatic (target and value orientated, programmanagement based), which meets the typical requirements for describing the phenomena of subjective reality. Since this phenomenon of subjective reality $(A)$ is information about this subject, it has its own specific bearer (let us designate it as X), which according to neuroscience is a certain brain neurodynamic system. Thus, the phenomenon of subjective reality is necessarily connected with the corresponding brain process like any information and its bearer. Analysis shows that the relationship between $\mathrm{A}$ and $\mathrm{X}$ is not causal. This is a special kind of functional connection: A and X are simultaneous and based on one cause, they are in a relation of a one-to-one correspondence; $\mathrm{X}$ is the code representation of $\mathrm{A}$ (or, briefly, code of A). This kind of connection can be called

a code dependence. It is formed in either phylogenesis or ontogenesis of a self-organizing system and is its functional element. A thorough investigation of the connection between $\mathrm{A}$ and $\mathrm{X}$, of the structural and functional organization of systems X-type means of desiphering the brain code of this phenomenon subjective reality. This is a special cognitive task, including the hermeneutic aspect, the phenomenon of understanding information embodied in one or another bearer.

With relation to these statements have been provided:

a) A theoretical and methodological analysis of decoding procedure, encoding and decoding processes, 
b) Discretization and quantification methods of the subjective reality continuum,

c) The formation of personal and interpersonal invariants of the selected phenomena of subjective reality as objects of neuroscientific research,

d) Typology of codes and relation of code structures of conscious and unconscious processes,

e) The connection and the difference between the detected neurodynamic corelate of a certain mental phenomenon and its actual code organization,

f) The hypothesis on the reasons of appearance of the quality of subjective reality in the course of evolution.

(These questions and a detailed justification of my theory are contained in my works: [6-8]).

Modern neuroscience has achieved significant success in deciphering the brain codes of subjective reality. For example, their result being a visual image experienced by a human is reproduced on a computer screen; brain neurodynamic correlates of many other psychic phenomena have been established [9-12]. "Brain reading", as one of the important areas of modern neuroscience, is developing successfully. Here, of course, a lot of debatable questions arise that concern the study of consciousness and require a thorough discussion. I would like to focus on the most important issue of these discussions - the question of the possibility of change the negative properties of consciousness. If the phenomenon of subjective reality and its neurodynamic bearer have the same cause, are simultaneous and are in one-to-one correspondence, that means that when I myself control this phenomenon of subjective reality - I am also controlling with my own will its neurodynamic bearer. Each of us has the ability to manage a certain class of his or her own brain neurodynamic systems, and at the same time manage the energy supply of these operations (the ability of mental self-determination). And everyone does it all the time, often not very well.

This determines the ability to arbitrarily control not only mental and bodily movements but also certain vegetative processes (as yogis do), and moreover, the ability to achieve changes at the genetic level, as recent studies show. All this points to extremely large, still weakly used resources of psychic self-regulation and self-organization. From this perspective, there are opportunities for deeper research into the phenomena of "tension of thought", "tension of will", intensification of the creative process and meditative practices, for creation of new effective ways of selfregulation, not only functional but also moral. The problem of neutralization, and, in the long run, overcoming negative properties of consciousness, involves extensive use of the richest phenomenological and meditative experience of Eastern philosophy and culture. Of course, productive concepts of Western philosophy and psychology are also important for this purpose. However, many hopes are associated with the development of neuroscience, which gradually clarifies certain brain functional structures responsible for altruistic and selfish intentions, allows deeper understanding of the phenomenon of aggression. Studies in this direction are very diverse. I want to cite, as an example, research in the field of mirror systems which I have already mentioned. They reveal the brain mechanisms of understanding another people's emotions, of sympathy and compassion manifestations. As Jacomo Rizzolatti and Corrao Sinigaglia write, "the ability of the brain to reflect the perception of the faces and gestures of other people and instantly encode them in terms of visceromotor reactions provides a brain substrate for empathy and compassion that regulates and guides our behavior and relationships at various levels and in various ways" [13]. Certainly, research in this area and the creation of means for changing consciousness should be carried out under strict social and humanitarian control.

Recently, neuroscience has been enriched with much more effective methods of visualization and mapping of brain processes. Among them are methods of optochemistry and optogenetics, neuroradiology or methods that allow penetrating into deep brain structures noninvasively, through the skull bones, using the effect of light passing through solids, etc. This opens a new stage in neuroscience research, the development of their new fruitful relationships with the achievements of artificial intelligence and information technology, moreover, with the whole system of convergent development of the NBIC (nanotechnology, biotechnology, information, cognitive technology and the corresponding areas of scientific knowledge). All this gives hope and some reasons to optimistically assess the possibility of goal-seeking changes of those extremely persistent dispositional structures of consciousness that determine such negative qualities as irrepressible consumerism, aggressiveness and extreme egoism. We can assume that this is the main problem of consciousness of our age. The future of the human civilization depends on its solution.

\section{References}

1. William J Ripple, Christopher Wolf, Thomas M Newsome, Mauro Galetti, Mohammed Alamgir, et al. (2017) World Scientist's Warning to Humanity: A Second Notice. Bio Science 67(12): 1026-1028.

2. Von Weizsaecker E, Wijkman A (2018) Come On! Capitalism, Shorttermism, Population and the Destruction of the Planet. Springer pp. 220.

3. Ramachandran VS (2011) The Tell-Tale Brain. A neuroscientist's Quest for What makes Us Human, New York, London.

4. Dubrovsky DI (2015) Subjective Reality and the Brain. To the question of the half-century experience of working out the difficult problem of consciousness in analytic philosophy. In: Dubrovsky DI (Eds.), The Problem Consciousness and the brain: Theoretical solution. Moscow, Russia pp. 66-154.

5. Dubrovsky DI (1971) Psychic Phenomena and the Brain: A Philosophical Analysis of the Problem in relation to certain topical problems of Neurophysiology, Psychology, and Cybernetics pp. 386.

6. Dubrovsky DI (2015) The Problem Consciousness and the brain: Theoretical solution pp. 208. 
7. Dubrovsky DI (2019) The problem of free will and modern neuroscience. Neuroscience and Behavioral Physiology 49(5): 629-639.

8. Dubrovsky David I (2019) The Hard Problem of Consciousness Theoretical solution of its main questions. AIMS Neuroscience 6(2): 85 103.

9. Miyawaki Yoichi, Hajime Uchida, Okito Yamashita, Hiroki C Tanabe, Norihiro Sadato, et al. (2008) Visual Image Reconstruction from Human Brain Activity using a Combination of Multiscale Local Image Decoders. Neuron 60(5): 915-929.

10. Nishimoto Sh, An T Vu, Thomas Naselaris, Yuval Benjamini, Bin Yu, et al. (2011) Reconstructing Neuron Visual Experience from Brain Activity Evoked by Natural Movies. Current Biology 21(19): 1641-1646.

ISSN: 2574-1241

DOI: 10.26717/BJSTR.2019.21.003565

David I Dubrovsky. Biomed J Sci \& Tech Res

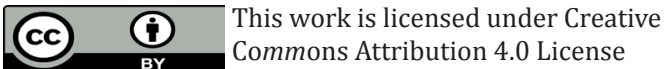

Submission Link: https://biomedres.us/submit-manuscript.php
11. Vu AT, Gallant JL (2015) Using a novel source-localized phase regressor technique for evaluation of the vascular contribution to semantic category area localization in BOLD fMRI. Frontiers in Neuroscience 9: 411.

12. Naselaris Nunez Eliza, Olman CA (2019) Voxelwise encoding models with non-spherical multivariate normal priors. Neuroimage 197: 482492.

13. Rizzolatti Giacomo, Sinigaglia Corrado (2012) Mirrors in the Brain. On the mechanisms of joint action and compassion. Moscow Languages of Slavic Cultures.

$\begin{array}{ll}\text { BIOMEDICAL } & \text { Assets of Publishing with us } \\ \text { RESEARCHES } & \text { - Global archiving of articles } \\ & \text { - Immediate, unrestricted online access } \\ & \text { - Rigorous Peer Review Process } \\ \end{array}$

